\title{
A numerical investigation of unsteady bubbly cavitating nozzle flows
}

\author{
A. T. Preston, T. Colonius, ${ }^{\text {a) }}$ and C. E. Brennen \\ Division of Engineering and Applied Science, California Institute of Technology, Pasadena, California 91125
}

(Received 27 June 2000; accepted 28 August 2001)

\begin{abstract}
The effects of unsteady bubble dynamics on cavitating flow through a converging-diverging nozzle are investigated numerically. A continuum model that couples the Rayleigh-Plesset equation with the continuity and momentum equations is used to formulate unsteady, quasi-one-dimensional partial differential equations. Flow regimes studied include those where steady-state solutions exist, and those where steady-state solutions diverge at the so-called flashing instability. These latter flows consist of unsteady bubbly shock waves traveling downstream in the diverging section of the nozzle. An approximate analytical expression is developed to predict the critical backpressure for choked flow. The results agree with previous barotropic models for those flows where bubble dynamics are not important, but show that in many instances the neglect of bubble dynamics cannot be justified. Finally the computations show reasonable agreement with an experiment that measures the spatial variation of pressure, velocity and void fraction for steady shockfree flows, and good agreement with an experiment that measures the throat pressure and shock position for flows with bubbly shocks. In the model, damping of the bubble radial motion is restricted to a simple "effective" viscosity, but many features of the flow are shown to be independent of the specific damping mechanism. () 2002 American Institute of Physics. [DOI: 10.1063/1.1416497]
\end{abstract}

\section{INTRODUCTION}

The first model of two phase flow through a convergingdiverging nozzle was proposed by Tangren et al. ${ }^{1}$ They employed a barotropic relation, which assumes that the fluid pressure is a function of fluid density only. This implies that the only effect of the disperse gas phase is to allow fluid compressibility which results in the bubbly mixture being treated as a single-phase compressible fluid. Brennen ${ }^{2}$ provides a general discussion of the barotropic model, as well as a summary of the work of Tangren et al.

Bubble dynamics are neglected by the barotropic model, but are thought to significantly alter the flow in cavitating nozzles, even in the mean. Wang and Brennen ${ }^{3}$ applied a nonlinear continuum bubbly mixture model ${ }^{4-6}$ to the computation of the steady flow through a converging-diverging nozzle. This model incorporates the Rayleigh-Plesset equation to predict bubble size and growth as a function of position and time. Wang and Brennen found two different steady state flow regimes to exist, and termed them quasi-steady and quasi-unsteady. The former is characterized by bubble growth that is induced by the low pressure region in the nozzle contraction, followed by a series of bubble collapses and rebounds downstream of the contraction. The quasiunsteady solutions correspond to flashing flow. Varying the upstream conditions causes the flow to bifurcate from one regime to the other. The bifurcation of the steady state equations has been studied by Delale et $^{\mathrm{al}} .^{7}$

To illustrate the two regimes, the method of Wang and Brennen is applied to a nozzle with a gentle contraction, depicted in Fig. 1. Wang and Brennen investigated the bifur-

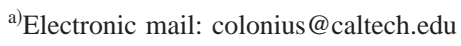

cation by varying the inlet void fraction; here we vary the cavitation number, $\sigma$, and achieve a similar result. Figure 1 presents the computed steady-state solutions for the pressure and bubble radius for cavitation numbers either side of the critical bifurcation value. The solid and dashed lines correspond, respectively, to the quasi-steady and quasi-unsteady solutions.

It is apparent in Fig. 1 that the flashing solution has unbounded bubble growth which results in a physically unrealistic downstream pressure. Physically realizable steadystate solutions do not exist in this flow regime, and hence an unsteady code is required to examine these flows. Ishii et al. ${ }^{8}$ proposed an unsteady bubbly flow model for the study of flows through a convergent-divergent nozzle. However, by assuming that the pressure inside the bubbles is equal to the ambient fluid pressure, they neglected the bubble dynamics which are important in the cavitating nozzle flow. Chen and Heister ${ }^{9}$ incorporated bubble dynamics into the computation of bubbly flow through a nozzle by using a form of Rayleigh-Plesset equation that was modified to allow for high void fractions. ${ }^{10}$ However, the nozzle flow was not the focus of their work and was only studied in noncavitating regimes for the purpose of demonstrating grid convergence.

The motivation of the present work is to investigate the cavitating regimes where steady-state computations predict a physically unrealizable flashing solution. An unsteady quasione-dimensional code, based on the bubbly flow model used by Wang and Brennen, is employed to demonstrate that physically realizable solutions in the flashing regime involve unsteady bubbly shock waves propagating downstream from the nozzle contraction. An important restriction of the present results is that an ad hoc, but computationally fast, model is used for damping the bubble radial motion. In Sec. 

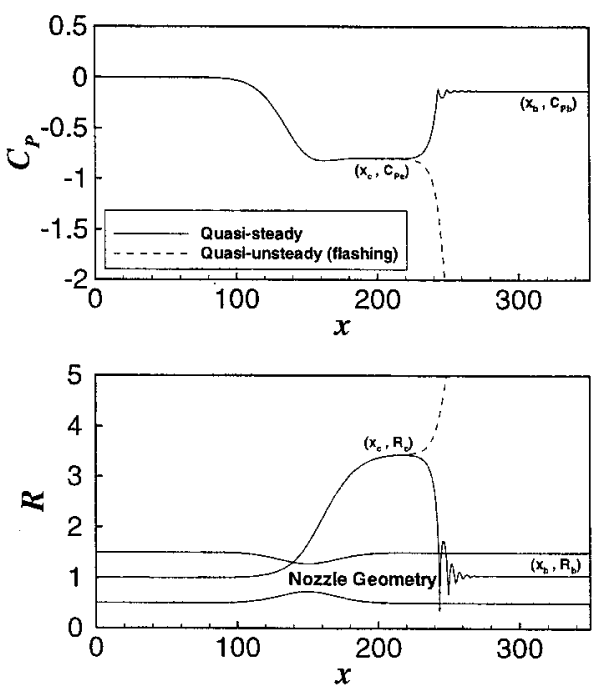

FIG. 1. The pressure coefficient and bubble radius for two steady computations. Solid line is quasi-steady $\left(\sigma=\sigma_{\text {crit }}^{+}\right.$), dashed line is quasi-unsteady or flashing $\left(\sigma=\sigma_{\text {crit }}^{-}\right)$. The cavitation number is defined as, $\sigma=\left(p_{0}^{\prime}\right.$ $\left.-p_{v}^{\prime}\right) / \frac{1}{2} \rho_{L}^{\prime} u_{0}^{\prime 2}$, where $p_{v}^{\prime}$ is the vapor pressure of the liquid, $p_{0}^{\prime}$ and $u_{0}^{\prime}$ are the upstream pressure and velocity, and $\rho_{L}^{\prime}$ is the liquid density.

III D we demonstrate that the basic flow features are independent of the damping mechanisms.

\section{THE MODEL AND NUMERICAL METHOD}

We consider a homogeneous continuum bubbly mixture model that was first proposed by van Wijngaarden., ${ }^{4,5}$ (Applying an order of magnitude analysis ${ }^{2}$ indicates that, for the present nozzle flows, relative motion of the two phases can be neglected.) The model starts with the conventional quasione-dimensional continuity and momentum equations for flow of a compressible fluid through a nozzle

$$
\begin{aligned}
& \frac{\partial}{\partial t}(\rho A)+\frac{\partial}{\partial x}(\rho u A)=0, \\
& \rho \frac{D u}{D t}+\frac{1}{2} \frac{\partial C_{P}}{\partial x}=0 .
\end{aligned}
$$

Note that the viscous terms and gravity have been neglected in the momentum equation. The two-phase bubbly mixture is now assumed to be made up of an incompressible liquid phase with a dilute gas phase consisting of many spherical bubbles. By assuming that the flow properties vary on length scales that are large compared to the bubble radius and noting that the gas phase is restricted to being dilute we can, at any point in the flow, relate the local bubble radius to the local pressure by the Rayleigh-Plesset equation

$$
\begin{gathered}
R \frac{D^{2} R}{D t^{2}}+\frac{3}{2}\left(\frac{D R}{D t}\right)^{2}+\delta_{D} \frac{1}{R} \frac{D R}{D t}+\frac{2}{W e}\left[R^{-1}-R^{-3 k}\right] \\
+\frac{\sigma}{2}\left[1-R^{-3 k}\right]+\frac{C_{P}}{2}=0 .
\end{gathered}
$$

The bubble population per unit liquid volume is fixed (neither fission nor fusion occur), so that the following relation between the mixture density and bubble radius holds

$$
\rho=\left[1+\frac{\alpha_{0} R^{3}}{1-\alpha_{0}}\right]^{-1},
$$

where $\alpha_{0}$ is the initial volume void fraction. Equations (3) and (4) dynamically relate the mixture density to the mixture pressure. These two equations effectively replace the usual barotropic relation used for single phase flows, and allow the set of equations to be closed.

In Eqs. (1)-(4) $\rho$ is the mixture density made dimensionless by the constant liquid density, $\rho_{L}^{\prime}$. The length and velocity scales used for nondimensionalization are the equilibrium bubble radius, $R_{0}^{\prime}$, and the nozzle inlet velocity, $u_{0}^{\prime}$. The pressure coefficient is defined as $C_{P}=\left(p^{\prime}\right.$ $\left.-p_{0}^{\prime}\right) / \frac{1}{2} \rho_{L}^{\prime} u_{0}^{\prime 2}$, where $p_{0}^{\prime}$ is the upstream pressure. The cavitation number is defined as $\sigma=\left(p_{0}^{\prime}-p_{v}^{\prime}\right) / \frac{1}{2} \rho_{L}^{\prime} u_{0}^{\prime 2}$, where $p_{v}^{\prime}$ is the vapor pressure of the liquid. The Weber number is given by $W e=\rho_{L}^{\prime} R_{0}^{\prime} u_{0}^{\prime 2} / S^{\prime}$, where $S^{\prime}$ is the (constant) surface tension. The use of the polytropic index, $k$, which is either unity for isothermal flow or the ratio of the specific heats of the gas phase for adiabatic flow, assumes that the bubble contents are fully mixed. This assumption combined with the use of an effective damping, $\delta_{D}$, to account for both viscous and diffusive contributions to damping of bubble radial motions, circumvents the need for solving unsteady diffusion equations at the scale of the bubble at every point in the flow. The impact of this simplified model is discussed in Sec. III D. The nozzle area, $A$, is nondimensionalized by the inlet area, $A_{0}^{\prime}$. Since viscous terms are neglected, $A_{0}^{\prime}$ does not appear as a parameter of the computation; only the axial area variation of the nozzle is relevent. Parenthetically, for comparisons to real experimental nozzles it is required that $\sqrt{A_{\min }^{\prime}} \gg R_{\max }^{\prime}$ for the continuum approximation to hold.

Equations (1)-(4) are integrated using a onedimensional Lagrangian finite volume scheme. This formulation allows the substantial derivatives to be treated as ordinary derivatives, and hence the Rayleigh-Plesset equation can be integrated as an ordinary differential equation (ODE). Consider a quasi-one-dimensional nozzle divided longitudinally into $N-1$ control volumes. Denoting the position of the control volume faces as $x_{j}(j=1,2, \ldots, N)$, we can define the nozzle areas and their derivatives at these positions:

$$
\begin{aligned}
& A_{j}=A\left(x_{j}\right), \\
& \frac{d A_{j}}{d x}=\frac{d A}{d x}\left(x_{j}\right),
\end{aligned}
$$

where $A(x)$ and $d A(x) / d x$ are known functions. Each control volume face moves at the local fluid velocity and, therefore,

$$
\frac{d x_{j}}{d t}=u_{j}
$$

where $u_{j}$ is shorthand for $u\left(x_{j}(t), t\right)$. Integrating Eqs. (1) and (2) over the control volumes we obtain, for $j=1,2, \ldots, N-1$

$$
\frac{d}{d t} \int_{x_{j}}^{x_{j+1}} \rho A d x=0,
$$




$$
\begin{aligned}
2 \frac{d}{d t} \int_{x_{j}}^{x_{j+1}} \rho u A d x= & A_{j} C_{P_{j}}-A_{j+1} C_{P_{j+1}} \\
& +\int_{x_{j}}^{x_{j+1}} C_{P} \frac{d A}{d x} d x .
\end{aligned}
$$

Equations (8) and (9), respectively, describe the rate of change of the total mass and momentum in the $j$ th control volume. Also, Eq. (3) can be split into two first order equations at each face $(j=1,2, \ldots, N)$

$$
\begin{aligned}
& \frac{d R_{j} V_{j}}{d t}+G_{j}+\frac{C_{P_{j}}}{2}=0, \\
& \frac{d R_{j}}{d t}-V_{j}=0,
\end{aligned}
$$

where

$$
G_{j}=\frac{V_{j}^{2}}{2}+\delta_{D} \frac{V_{j}}{R_{j}}+\frac{2}{W e}\left[R_{j}^{-1}-R_{j}^{-3 k}\right]+\frac{\sigma}{2}\left[1-R_{j}^{-3 k}\right] .
$$

Finally, the density and bubble radius at the faces are related by

$$
\rho_{j}=\left[1+\frac{\alpha_{0} R_{j}^{3}}{1-\alpha_{0}}\right]^{-1} .
$$

To integrate this system of (as yet exact) equations, it remains to approximate the integrals in Eqs. (8) and (9). A second-order approximation is used

$$
\int_{x_{j}}^{x_{j+1}} f d x=\frac{\Delta x_{j}}{2}\left(f_{j}+f_{j+1}\right) O\left(\Delta^{3}\right),
$$

where $\Delta x_{j} \equiv x_{j+1}-x_{j}$, and $f$ is any of $\rho A, \rho u A$, or $C_{P}(d A / d x)$.

Equations (5)-(13) are $8 N-2$ ODEs for $8 N$ unknowns $\left(\rho_{j}, R_{j}, V_{j}, C_{P_{j}}, u_{j}, A_{j}, d A / d x_{j}\right.$, and $x_{j}$ at the edges of the control volumes, $j=1,2, \ldots, N)$. Specifying both of the boundary pressures, $C_{P_{1}}$ and $C_{P_{N}}$ closes the system. Alternative boundary conditions, such as the nonreflective boundary condition developed by Colonius et al. ${ }^{11,12}$ and the impedance boundary condition used later in this paper, have also been successfully implemented.

The equations are solved in the Lagrangian coordinate system, whereas the nozzle boundary conditions should be implemented in an Eulerian coordinate system. To circumvent this situation a special control volume with a fixed upstream face and a moving downstream face is used at the upstream boundary. Hence we replace Eq. (7) for the $j=1$ case with $x_{1}=$ constant. Additional flux terms are also added to Eqs. (8)-(11). It is clear that the control volume will become very large as the downstream face is convected away from the stationary upstream face. Remeshing is required to ensure that the accuracy of Eq. (14) is maintained. This is achieved by simply splitting the control volume into two as necessary as the computation proceeds. Maintaining consistency with the order of approximation of Eq. (14), variable values at the new face are obtained by linearly interpolating from values at either side. As a new control volume is cre- ated at the upstream boundary a control volume is removed from the downstream boundary. Hence the downstream boundary is only approximately fixed in position, with fluctuations caused by the truncations as well as net expansions or compressions of the fluid over the entire domain. In practice the downstream boundary is positioned far enough from the nozzle contraction that, after initial transients, there are no appreciable gradients in the solution near the boundary, so that the exact location of the boundary is not important.

The discretized equations have similar properties to those arising in earlier work ${ }^{11,12}$ that examined the generation of bubbly shocks by an oscillating plane boundary. That is, they are stiff, and do not conserve mass precisely when an explicit time marching scheme is used. Hence an implicit Euler method is used for the basic time advancement. This is combined with a Richardson extrapolation method. ${ }^{13,14}$ The basic premise of the method is to compute a series of predictions for the solution at the new time level based on different numbers of subdivisions of the time interval. The series of predictions is then used to extrapolate to the limit of zero time step, and to provide an error estimate for the integration. The overall time step is adjusted based on the number of subdivisions and the error estimate.

The basic time advancement of the extrapolation method is the implicit Euler method. Using the integration scheme on Eqs. (7)-(11) and going through the algebra, we can reduce the number of independent variables to $2 N$ resulting in equations of the form,

$$
F_{j}\left(X_{k}^{n+1}\right)=0, \quad j=1, \ldots, 2 N, \quad k=1, \ldots, 2 N,
$$

where $\boldsymbol{X} \equiv\left[u_{1}, R_{1}, x_{2}, R_{2}, x_{3}, R_{3}, \ldots, x_{N}, R_{N}\right]$. In each equation $F_{j}$, various parameters of the problem also appear as well as the fields from previous time levels. Newton's method is used to solve the nonlinear equations. The system of Eqs. (15) results in a six-banded Jacobian matrix, enabling relatively efficient solution.

Grid convergence studies were conducted for flows in the regime where a steady bubbly shock wave stands in the diverging portion of the nozzle. For each of the three different grid resolutions the back pressure was lowered to initiate the flow, and the computation was performed until steady state was reached. Figure 2 presents the computed bubble radius for the different grid resolutions. The medium and fine grids are indistinguable indicating that the solution is meshindependent. The coarse grid is slightly different because it is not quite fine enough to fully resolve the series of bubble rebounds and collapses following the bubbly shock. In the remainder of the paper all results presented have enough grid points to fully resolve the bubbly shock structure.

\section{RESULTS}

The nondimensional parameters that are chosen to be studied are $\alpha_{0}=10^{-2}, W e=117, \delta_{D}=0.43, k=1.4$ (adiabatic) and $\sigma$ ranging from about 0.93 to 1.20 . These correspond to atmospheric pressure at the nozzle inlet $\left(p_{0}^{\prime}\right.$ $=101.3 \mathrm{kPa})$ with water at $20^{\circ} \mathrm{C}\left(\rho_{L}^{\prime}=1000 \mathrm{~kg} / \mathrm{m}^{3}, S^{\prime}\right.$ $\left.=0.073 \mathrm{~N} / \mathrm{m}, p_{v}^{\prime}=3.5 \mathrm{kPa}\right)$ and air bubbles of equilibrium radius, $R_{0}^{\prime}=10^{-4} \mathrm{~m}$, with inlet velocity, $u_{0}^{\prime}$, ranging from 


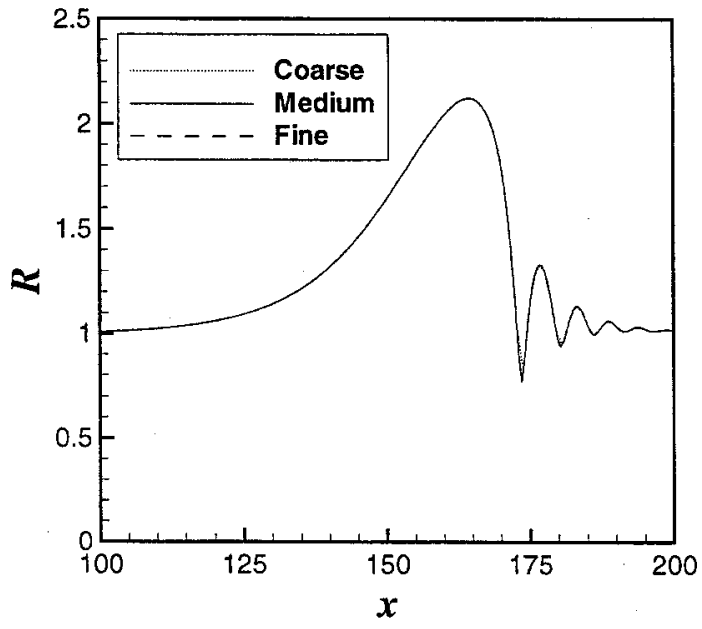

FIG. 2. The bubble radius for three different grid resolutions.

12.7 to $14.5 \mathrm{~m} / \mathrm{s}$. The value of $\delta_{D}$ is chosen to achieve realistic solutions with only a few bubble rebounds. It is shown later that for an effective damping less than about 0.5 the macroscopic flow properties are independent of the effective damping. The nozzle has a Gaussian area variation given by

$$
A(x)=1-\left(1-A_{\min }\right) e^{\left.-\left[\left(x-x_{0}\right) / w\right)\right]^{2}},
$$

and for the present study we focus on the values $A_{\text {min }}$ $=0.75, x_{0}=150.0, w=30.0$.

\section{A. Flow regimes}

A series of computations are performed where the back pressure is varied over a wide range. As the back pressure is changed we obtain different mass flow rates through the nozzle, and hence the cavitation number is also varying.

The computed pressure, bubble radius and flow velocity for a typical set of computations are presented in Fig. 3. The solid lines represent final steady-state solutions (obtained by computing until steady state is reached), while the dashed lines represent instantaneous flowfields as an unsteady shock wave travels downstream through the nozzle.

It is seen that much like the quasi-one-dimensional nozzle flows for a perfect gas, different regimes exist depending upon the value of the back pressure (or cavitation number). These regimes are:

$$
\text { Steady solution with no shocks }\left(0>C_{P b}>C_{P_{\text {crit }_{1}}}\right)
$$

Recall that for any inviscid flow in a nozzle with equal inlet and outlet areas (such as the one being examined) that no steady state, shockfree solutions exist for a nonzero pressure drop. If a pressure drop is applied the flow will accelerate until it becomes choked at the throat. A shock will then form in the diverging section of the nozzle, with its position depending upon the value of the pressure drop. The entire total enthalpy drop is achieved over the shock, with all other parts of the flow remaining isentropic.

In the present bubbly model there is no macroscopic viscosity of the fluid, but there are losses associated with the bubble dynamics. These are accounted
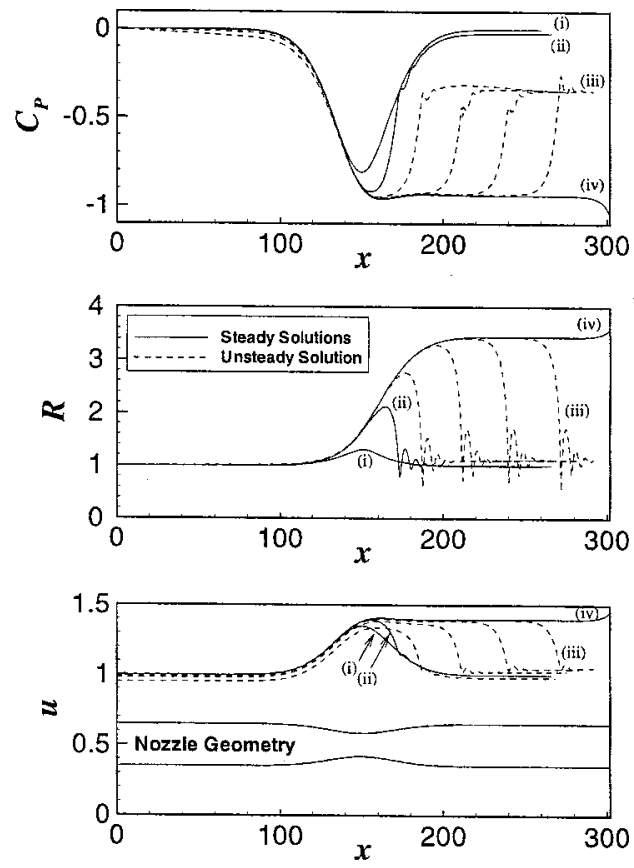

FIG. 3. The pressure coefficient, bubble radius and flow velocity for four different back pressures (and cavitation numbers). (i) Steady-state solution with no shocks $(\sigma=1.20)$. (ii) Shock standing in diverging section of nozzle $(\sigma=0.940)$. (iii) Unsteady shock traveling down nozzle $(\sigma=0.932)$. (iv) Steady-state solution with expansion near nozzle exit ( $\sigma=0.937)$. (All computations have $\alpha_{0}=10^{-2}, W e=117, \delta_{D}=0.43$.)

for by the effective damping, $\delta_{D}$, in the RayleighPlesset equation. Provided that the effective damping is not zero, then for a small pressure drop a steady state, shockfree solution is obtained. One such solution is plotted as curve (i) in Fig. 3. It is apparent that there is only small growth of the bubble radius, and no collapses and rebounds.

(ii) Stationary shock in diverging section of nozzle $\left(C_{\text {crit }_{1}}>C_{P b}>C_{P_{\text {crit }_{2}}}\right)$

The pressure drop is now large enough to cause choking at the throat and the formation of a steady bubbly shock wave in the diverging section of the nozzle. Curve (ii) represents one such solution. The bubbly shock structure is most apparent in the graph of the bubble radius, which shows the characteristic bubble growth followed by a succession of collapses and rebounds. The pressure in this case also exhibits a relatively sharp recovery associated with the bubbly shock wave.

It would be expected that the shock position would be a function of the back pressure in a manner analogous to the gas dynamics case. This is shown to be the case in Sec. V B, where the computed shock position is compared with experimentally observed shock positions.

(iii) Unsteady shock traveling down nozzle $\left(C_{\text {crit }_{2}}>C_{P b}\right.$ $\lesssim-\sigma)$

The pressure drop is now large enough to cause the bubbly shock wave to move out of the diverging sec- 
tion and propagate downstream. The dashed curves of Fig. 3 show the solution at four different times. The time interval between each curve is constant, so it is evident that the propagation speed of the bubbly shock is approximately constant.

It is also interesting to note that while the structure of the shock in terms of the bubble radius and flow velocity remains essentially the same at each time instant, the structure in terms of the pressure does not. This is apparent in the last of the instantaneous curves which shows a larger pressure peak at the position of first collapse. In actuality similar pressure peaks manifest themselves at other earlier instances in the computation, but by coincidence the time instances shown on the plot do not exhibit these. Studying similar plots with a far higher temporal resolution indicates that the magnitude of the pressure peak at first collapse in fact oscillates in time, probably due to acoustic waves reflecting between the shock and the boundary. The pressure is far more sensitive to these waves than either the bubble radius or flow velocity.

Given the upstream and downstream radius, as well as the upstream pressure, Eq. (6.69) of Brennen ${ }^{2}$ can be used to compute the speed of the one-dimensional shock. This was done for the case illustrated with favorable comparisons. However, the computed shockspeed is very sensitive to the value of upstream pressure that is used; indeed it is possible compute a shock speed of zero with only the smallest of changes to the upstream pressure.

It is likely that within this range of back pressures it is physically possible to have a final steady-state solution where the flow in the nozzle is "overexpanded," and the increase to the back pressure takes place across a system of compressions and expansions outside the nozzle.

(iv) Steady, underexpanded flow $\left(C_{P b} \lesssim-\sigma\right)$

Now the back pressure is low enough to allow the shock to pass through the downstream boundary and out of the computational domain. The flow in the nozzle is "underexpanded" and expands near the end of the nozzle to match the back pressure. This is apparent in curve (iv) of the pressure plot in Fig. 3, which shows the expansion taking place near the domain boundary. The flashing solution of Fig. 1 is one of these solutions, albeit with an unrealistically low back pressure.

If we choose the back pressure to match the pressure upstream of the shock wave (i.e., $C_{P b} \approx-\sigma$ ), then there would be no such expansion. This corresponds to the "perfectly expanded" solution of the flow of a perfect gas.

Calculations with a lower void fraction of $\alpha_{0}=10^{-3}$ were performed to evaluate the effect of void fraction on the various regimes discussed above. Figure 4 presents the bubble radius for this series of computations. Once again the solid lines correspond to steady solutions while the dashed lines represent a time series of the unsteady solution. The

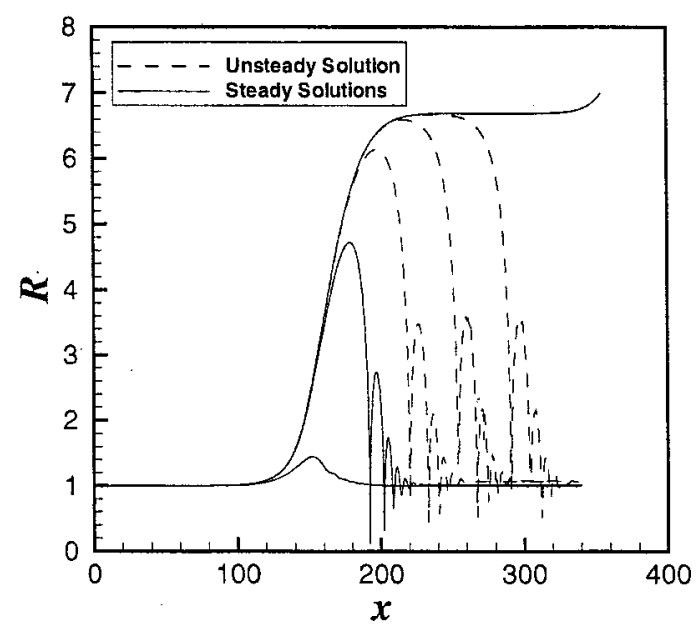

FIG. 4. The bubble radius for a set of calculations with lower void fraction $\left(\alpha_{0}=10^{-3}, \sigma=0.688\right.$ to $\left.1.000, W e=159, \delta_{D}=0.37\right)$.

behavior is qualitatively similar to that of the higher void fraction computations. As expected, the lower void fraction results in higher maximum bubble radius and hence more violent collapses.

\section{B. Choking}

As the cavitation number is decreased, the back pressure will also decrease naturally until, eventually, the flow becomes choked. The decrease of the cavitation number can be considered as a combination of two physical changes to the flow; decreasing the inlet pressure, $p_{0}^{\prime}$ and/or increasing the inlet velocity, $u_{0}^{\prime}$. If we consider situations where the inlet pressure is fixed then only the inlet velocity is changing, and from the definition of the cavitation number

$$
u_{0}^{\prime}=\left[\frac{p_{0}^{\prime}-p_{v}^{\prime}}{\frac{1}{2} \rho_{L}^{\prime} \sigma}\right]^{1 / 2}
$$

The dimensional mass flow rate is given by

$$
\dot{m}^{\prime}=\rho_{0}^{\prime} u_{0}^{\prime} A_{0}^{\prime}=\rho_{L}^{\prime}\left(1-\alpha_{0}\right) u_{0}^{\prime} A_{0}^{\prime} .
$$

Substituting Eq. (17) into Eq. (18), nondimensionalizing by the choked mass flow rate, and cancelling all the constant inlet conditions yields the following simple equation for the nondimensional mass flow rate

$$
\dot{m} \equiv \frac{\dot{m}^{\prime}}{\dot{m}_{\text {crit }}^{\prime}}=\left[\frac{\sigma_{\text {crit }}}{\sigma}\right]^{1 / 2},
$$

where $\sigma_{\text {crit }}$ is the cavitation number at choking.

Many calculations of the steady flow solution were carried out with varying cavitation numbers. Figure 5 plots the nondimensional mass flow rate [computed by Eq. (19)] versus the back pressure which is obtained as a result of each calculation. Results with effective dampings ranging from 0.22 to 0.85 are shown. It is interesting to note that the variation of effective damping does not affect the critical choking 


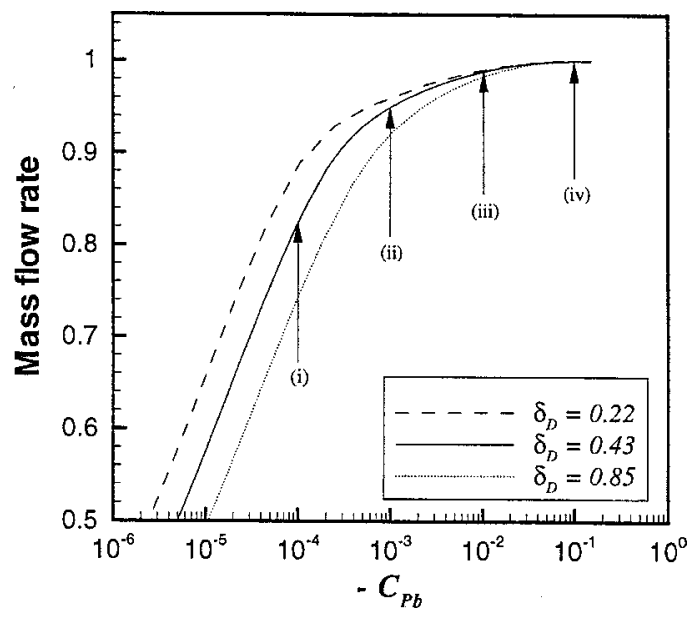

FIG. 5. Nondimensional mass flow rate as a function of back pressure coefficient for different values of effective damping, $\delta_{D}$, in the range 0.22 to $3.78\left(\alpha_{0}=10^{-2}, W e=117\right)$.

back pressure. This is discussed further in Sec. III C. As expected, for a given back pressure, cases with a smaller effective damping have a larger mass flowrate.

Figure 6 presents the bubble radius for four different back pressures indicated by (i)-(iv) in Fig. 5. Curve (i) in Fig. 6 illustrates that for small pressure drops there are no bubble collapses or rebounds. This accounts for the straight sections of the curves in Fig. 5. For larger pressure drops bubble collapses and rebounds become apparent [curve (ii) in Fig. 6]. This increases the losses in the system and hence causes the curved sections in Fig. 5. This smooth transition to choked flow continues as the pressure drops become larger and the bubble dynamics become more pronounced [curve (iii) in Fig. 6], until eventually the flow chokes and a bubbly shock wave forms [curve (iv) in Fig. 6].

\section{Critical back pressures}

For a particular set of flow parameters it would be useful to be able to predict the back pressure at which the flow

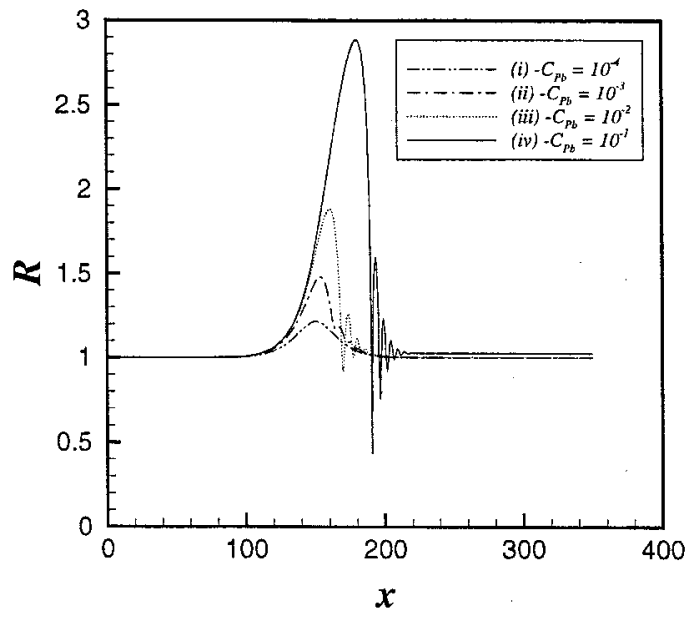

FIG. 6. The bubble radii corresponding to the four back pressures indicated in Fig. $5\left(\delta_{D}=0.43, \alpha_{0}=10^{-2}, W e=117\right)$. chokes and shocks form $\left(C_{P \text { crit }_{1}}\right)$, as well as the back pressure at which the shock becomes unsteady and begins traveling downstream through the constant area portion of the nozzle $\left(C_{P_{\text {crit }}}\right)$. The first transition is difficult to analyze since it is not clear when a series of bubble collapses and rebounds become a bubbly shock, and the finite thickness of a bubbly shock wave means that the nozzle area change that occurs over the shock thickness cannot be neglected. However, the second transition occurs in the constant area part of the nozzle and enables the one-dimensional jump conditions to be used to predict $C_{P_{\text {crit }}}$.

Consider the typical steady bubbly shock wave shown in Fig. 1. Integrating the steady forms of Eqs. (1) and (2) from the position of critical radius upstream of the shock $\left(x_{c}\right)$, to a position well downstream of the shock $\left(x_{b}\right)$ yields

$$
\begin{aligned}
& \left.\rho u A\right|_{x=x_{c}} ^{x=x_{b}}=0, \\
& \left.A\left(2 \rho u^{2}+C_{P}\right)\right|_{x=x_{c}} ^{x=x_{b}}-\int_{x_{c}}^{x_{b}} C_{P} \frac{d A}{d x} d x=0 .
\end{aligned}
$$

Also note that at positions $x_{c}$ and $x_{b}$ the derivatives with respect to $x$ vanish, so that for the steady flow the RayleighPlesset equation reduces to

$$
C_{P}\left(R_{c, b}\right)=-\sigma\left[1-R_{c, b}^{-3 k}\right]-\frac{4}{W e}\left[R_{c, b}^{-1}-R_{c, b}^{-3 k}\right] .
$$

Substituting Eq. (22) into (21) and noting that the integral term in Eq. (21) vanishes because there is no area change between $x_{c}$ and $x_{b}$, yields a nonlinear equation relating $R_{b}$ to $R_{c}$. Noticing that for the current computations $R_{b}=1+R_{b}^{\prime}$ where $R_{b}^{\prime} \ll 1$, it is useful to linearize this equation with respect to $R_{b}^{\prime}$. Neglecting terms higher than second order, allows $R_{b}^{\prime}$ to be determined as an explicit function of $R_{c}$

$$
R_{b}^{\prime}=\frac{h\left(R_{c}\right)-\rho_{0}}{3 \alpha_{0} h\left(R_{c}\right)-\omega_{N}^{2}},
$$

where

$$
h\left(R_{c}\right) \equiv \frac{C_{P}\left(R_{c}\right)}{2}+\rho\left(R_{c}\right) u_{c}^{2}=\frac{C_{P}\left(R_{c}\right)}{2}+\frac{\rho_{0}^{2}}{\rho\left(R_{c}\right)},
$$

and $\omega_{N}$ is the bubble natural frequency which is computed by

$$
\omega_{N}^{2} \equiv \frac{3 k \sigma}{2}+(3 k-1) \frac{2}{W e} .
$$

Once $R_{b}^{\prime}$ is computed, the following variables can also be computed to first order:

$$
\begin{aligned}
& R_{b}=1+R_{b}^{\prime}, \\
& C_{P_{b}}=-2 \omega_{N}^{2} R_{b}^{\prime}, \\
& u_{b}=1+3 \alpha_{0} R_{b}^{\prime} .
\end{aligned}
$$

Of course the present method requires knowledge of the critical radius $R_{c}$. It would be preferable to be able to predict the critical back pressure from knowledge of the flow parameters only. Wang and Brennen ${ }^{3}$ neglected the integral term of 
Eq. (21), and assumed that the critical bubble radius was large compared to the equilibrium radius to develop the simple approximate expression, $R_{c}=\left(\sigma / 2 \alpha_{0}\right)^{1 / 3}$. Applying the method to the computation of Fig. 1 where $\sigma_{\text {crit }}=0.932$ and $\alpha_{0}=10^{-2}$ yields $R_{c}=3.598$, and $C_{P b_{\text {crit }}}=-0.035$. This is vastly different from the back pressure in Fig. 1 which is -0.151 .

From the computation of Fig. 1 we find that the critical radius is in fact 3.429 which is approximately five percent lower than that predicted by the method of Wang and Brennen. Using this value or $R_{c}$ in Eqs. (23) and (25) yields $C_{P b_{\text {crit2 }}}=-0.160$, which compares well with the value in Fig. 1.

In order to obtain a reasonable estimate of the critical back pressure it is, therefore, crucial to first obtain an accurate estimate of the critical radius. The most significant source of error in Wang and Brennen's ${ }^{3}$ estimate of $R_{c}$ is the neglect of the integral term of Eq. (21) when integrating from the initial condition through the nozzle contraction to the position of critical radius. Wang ${ }^{15}$ addresses this issue and formulates a complicated nonlinear equation for $R_{c}$, which has to be solved numerically and is subject to a convergence condition. It is likely that this estimate would result in a better estimate of the critical back pressure.

It is important to note that the preceding analysis does not involve the effective damping. That is, the critical back pressure is independent of the effective damping used. This has already been observed in Sec. III B, where it was demonstrated that the onset of choking did not change when the effective damping was varied. The role of the effective damping is discussed more fully in the next section.

\section{Effect of damping}

The present model employs the use of an effective damping parameter to account for all radial damping mechanisms of the bubble motion. This is achieved by using a total effective liquid viscosity to include the contributions to damping from acoustic radiation and mass and thermal diffusion. van Wijngaarden ${ }^{5}$ reviews some analytical and empirical expressions for contributions to the total effective viscosity from viscous, acoustic, and thermal effects. These estimates are generally based on low amplitude linear motions, such as the attenuation of sound waves, whereas in the nozzle flow there is strong bubble growth and collapse. Recent studies ${ }^{16-18}$ have investigated the diffusive damping mechanism for noncondensible gas bubbles in the nonlinear regime by numerically solving the full unsteady diffusion equations at the scale of the bubble. They indicate that the simple model that we have used may not be able to correctly capture the structure of the bubbly shock waves. However, other important damping effects, such as acoustic radiation and bubble fission, have not yet been fully addressed in any of the studies.

Colonius et al. ${ }^{12}$ recently demonstrated that for lowfrequency forcing of bubbles it is relatively unimportant to model the detailed diffusive processes in the bubble. They demonstrated that the value of effective damping parameter used does not have a significant impact on the macroscopic

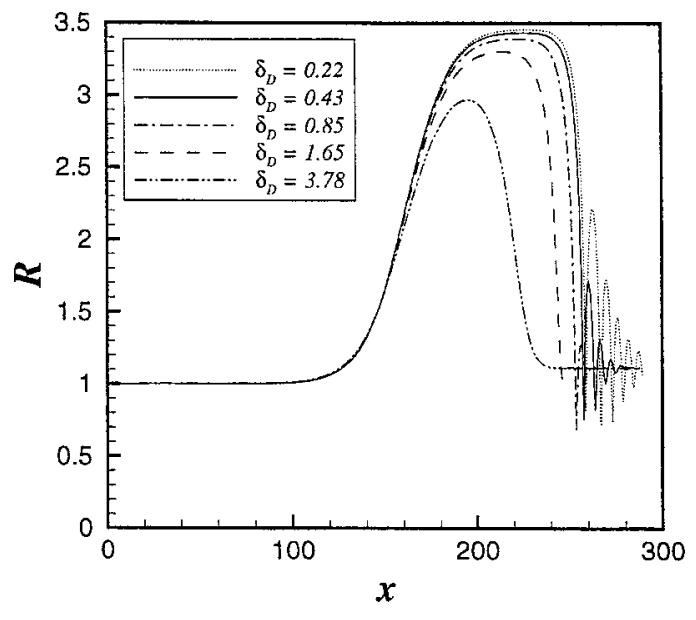

FIG. 7. Bubble radius for a series of different values of effective damping, $\delta_{D}$, in the range 0.22 to $3.78\left(\sigma=0.76\right.$ to $0.95,-C_{P_{b}}=0.27$ to $0.36, W e$ $=115$ to 153 ).

flow field as long as it is below a critical value. Their result hinged on the fact that the dissipation associated with the shock jump conditions is much larger than, and independent of, the dissipation provided by any of the damping processes that are modeled by the effective damping. For the present nozzle flow being studied the independence of the shock jump conditions on the value of the effective damping have been previously observed in Secs. III B and III C. Moreover, we now demonstrate that for realistic values of effective damping that the basic flow features are also independent of the magnitude of the effective damping parameter so long as it is sufficiently small (in the asymptotic sense). A series of unsteady computations with effective damping ranging from 0.22 to 3.78 was performed. The initial values of the other parameters were held constant, but the final values of the parameters varied slightly due to the renormalization with the inlet velocity. Figure 7 plots the bubble radius for each of these calculations at a time when the bubbly shock is propagating in the constant area section of the nozzle. For values of effective damping lower than about 0.5 the macroscopic behavior becomes independent of effective damping. That is, there is large growth of the bubble radius followed by a rapid collapse; the jump conditions across the shock are not significantly impacted by the value of effective damping. The main effect of decreasing the effective damping is to increase the amplitude and number of the bubble rebounds. For larger values of effective damping the bubble growth begins to be affected, eventually limiting the growth to the extent that there is no sharp collapse. For the results presented elsewhere in this paper the effective damping is chosen to be small enough so that the macroscopic flow properties are independent of decreases or small increases in the effective damping.

\section{COMPARISON TO BAROTROPIC MODEL}

Barotropic results for isothermal $(k=1)$ nozzle flow were first reported by Tangren et al. ${ }^{1}$ and differs from the present model in that bubble dynamic effects are neglected; at every point in the barotropic flow the bubbles are in equi- 
librium with the local pressure. Brennen ${ }^{2}$ presents the barotropic results for any polytropic index, and nondimensionalizing those equations for the case $k \neq 1$, and $u_{0}^{\prime} \neq 0$ yields the following set of equations:

$$
\begin{aligned}
& u=\frac{1-\alpha_{0}}{A(1-\alpha)}, \\
& u^{2}-1=\widetilde{\sigma}\left[1-r^{k}-\frac{k}{k-1} \frac{\alpha_{0}}{1-\alpha_{0}}\left\{1-r^{k-1}\right\}\right], \\
& C_{P}=\widetilde{\sigma}\left[r^{k}-1\right],
\end{aligned}
$$

where $r$ is defined as

$$
r \equiv \frac{\alpha_{0}(1-\alpha)}{\alpha\left(1-\alpha_{0}\right)},
$$

and $\widetilde{\sigma}=p_{0}^{\prime} / \frac{1}{2} \rho_{L}^{\prime} u_{0}^{\prime 2}$ is a parameter which is the same as the cavitation number if $p_{v}^{\prime}=0$. Equation (27) represents continuity, Eq. (28) is the momentum equation that has been integrated using the barotropic relation given by Eq. (29). Substituting Eq. (27) into Eq. (28) yields an algebraic equation that can be solved for $\alpha$ if the nozzle area and initial void fraction is specified. The flow velocity and pressure can then be computed from Eqs. (27) and (29), respectively.

Consider solving for $\alpha$ in the nozzle throat, where $A$ $=A_{\min }$. Equations (27) and (28) have either zero, one, or two real roots, depending upon the value of $\widetilde{\sigma}$. For high values of $\widetilde{\sigma}$ there exist two real roots, corresponding to subsonic and supersonic conditions. Given that the initial condition is subsonic, only the subsonic root is valid for these conditions. As $\widetilde{\sigma}$ is lowered the two roots approach each other until, for a particular critical value of $\widetilde{\sigma}$, there is only the single sonic solution at the throat, corresponding to choked flow. For this $\widetilde{\sigma}_{\text {crit }}$ the flow downstream of the throat can be either subsonic or supersonic, depending on the downstream boundary condition. For values of $\widetilde{\sigma}$ below $\widetilde{\sigma}_{\text {crit }}$, there are no solutions, indicating that no steady-state solutions exist.

It is useful to compare the results of the present paper (which we term the dynamic calculations) with those of the barotropic model. To compare the barotropic calculations we first proceed to find $\widetilde{\sigma}_{\text {crit }}$ by trial and error. That is, $\widetilde{\sigma}$ is varied until Eqs. (27) and (28) have only the sonic root at the throat. The value of $\widetilde{\sigma}_{\text {crit }}$ will depend only upon the initial void fraction, $\alpha_{0}$, and throat area, $A_{\min }$. For the computations of Fig. $3\left(\alpha_{0}=10^{-2}, A_{\min }=0.75\right)$ we find that $\widetilde{\sigma}_{\text {crit }}$ $=1.011$. This is different to the critical cavitation number in Fig. 3, which is $\widetilde{\sigma}_{\text {crit }}=0.937$. The difference of $\widetilde{\sigma}_{\text {crit }}-\sigma_{\text {crit }}$ $=1.011-0.937=0.074$ is due to the (constant) vapor pressure, $p_{v}^{\prime}$, of the liquid. It is chosen to keep this algebraic difference constant for comparisons at all values of $\sigma$. That is, to compare a barotropic calculation to a dynamic computation with a cavitation number of $\sigma$, we use $\widetilde{\sigma}=\sigma+0.074$.

The computed dynamic and barotropic pressures for three different cavitation numbers are presented in Fig. 8. The upper graph presents comparisons for a flow that is far from critical (high cavitation number). The curves for the dynamic and barotropic calculations overlay each other indicating that for flows which are far from critical bubble dynamics are not important and the barotropic model is valid.
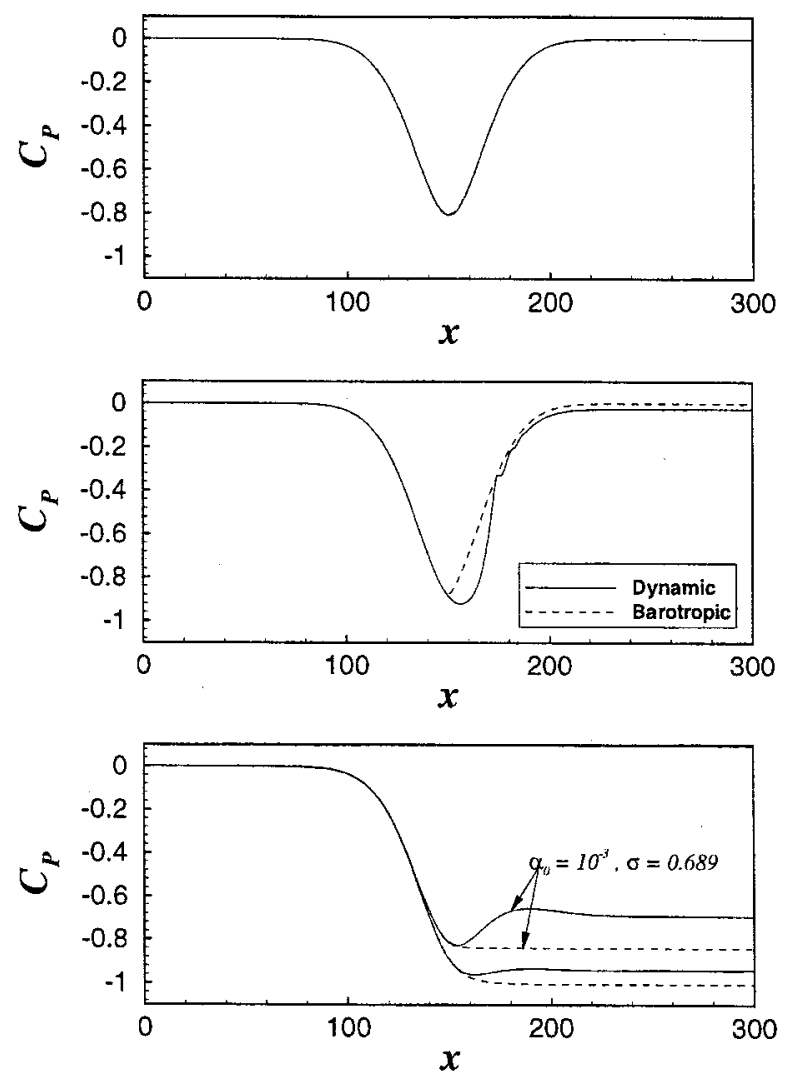

FIG. 8. Comparison of pressures for dynamic and barotropic computations for $\sigma=1.200$ (upper), $\sigma=0.940$ (middle), and $\sigma=0.937$ (lower) $\left(\alpha_{0}\right.$ $=10^{-2}$ unless otherwise specified).

The middle graph compares the two models for a flow that is almost critical (cavitation number only slightly above critical). The agreement is good up until the throat, at which point the dynamic computation develops an asymmetry due to the radial inertia associated with the bubble growth. It is apparent that the barotropic model is no longer valid, due to the effects of bubble dynamics.

The lower graph presents comparisons for computations at the critical condition. Agreement is excellent up until just after the throat, at which point the pressure in the dynamic computation increases above the minimum value attained near the nozzle throat. The minimum pressure in the throat being lower than the back pressure is again caused by the radial inertia that the bubbles have as they approach the throat. The barotropic model is unable to model this behavior due to the neglect of bubble dynamics. Also presented in the lower figure is a comparison at critical condition for a lower initial void fraction of $10^{-3}$. It is noted that the differences between the dynamic and barotropic models are greatest for the lower void fraction flow. This is consistent with the observation in Sec. III A that lower void fraction computations have a higher maximum bubble radius and more violent bubble collapses.

\section{COMPARISON TO EXPERIMENTS}

\section{A. Shockfree steady flow}

Here we will compare the results of the bubbly model with the experiments of Ishii et al. ${ }^{8}$ who measured the pres- 

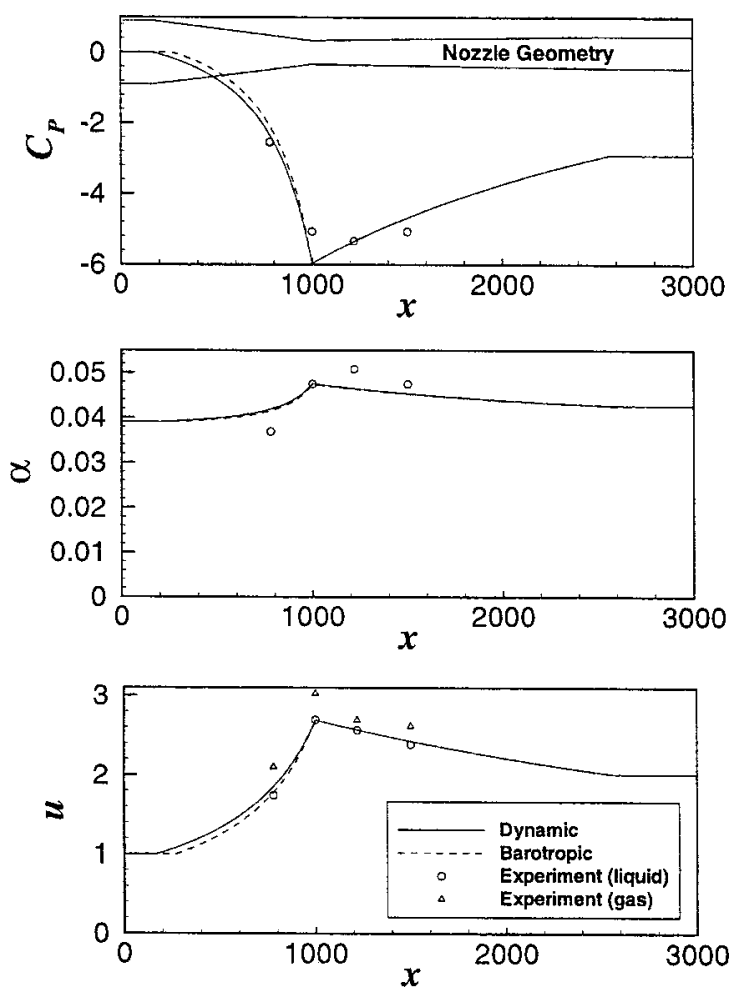

FIG. 9. Comparison of Ishii et al. experimentally measured pressures, void fractions and velocities with barotropic and computed solutions $\left(\alpha_{0}\right.$ $=0.039, \sigma=23.5, W e=20.7, \delta_{D}=0.5$ ).

sure, void fraction, and flow velocities of both the liquid and gas components at four locations in a steady nozzle flow, and compared them with their own bubbly flow model. Their model assumed that the pressure inside a bubble was equal to the ambient pressure, and hence neglected any of the bubble dynamics described by the Rayleigh-Plesset equation. They did, however, account for relative motion between the liquid and gas phases, which may be important to correctly predict the void fraction distribution in the nozzle.

The nozzle had an area that varied linearly to a throat with area ratio (relative to the inlet) of 0.375 , and then expanded to an area ratio of 0.50 at the exit. The flow conditions were water at $20^{\circ} \mathrm{C}\left(\rho_{L}^{\prime}=998 \mathrm{~kg} / \mathrm{m}^{3}, p_{v}^{\prime}=3.5 \mathrm{kPa}, S^{\prime}\right.$ $=0.073 \mathrm{~N} / \mathrm{m}$ ), with air bubbles with average equilibrium radius, $R_{0}^{\prime}=10^{-4} \mathrm{~m}$, with inlet velocity, $u_{0}^{\prime}=3.90 \mathrm{~m} / \mathrm{s}$, and inlet pressure, $p_{0}^{\prime}=182 \mathrm{kPa}$. These conditions resulted in a cavitation number, $\sigma=23.5$ and Weber number, $W e=20.7$. From the air and water mass flow rates that are provided, and assuming no relative motion at the inlet, it is possible to compute the inlet void fraction as approximately, $\alpha_{0}$ $=0.039$.

Since the experimental data are only for a steady flow, a steady code based on that of Wang and Brennen ${ }^{3}$ is used to compute the solution. In addition the barotropic solution discussed in Sec. IV is calculated. Figure 9 shows the comparisons of the dynamic computation (solid lines) and the barotropic calculation (dashed lines) to the experimentally measured pressures, void fractions and velocities of Ishii et al. ${ }^{8}$ The maximum bubble growth in this flow is only about 7 percent which results in $\sqrt{A_{\min }^{\prime}} / R_{\max }^{\prime}=124$, so the continuum approximation is valid. The small amount of bubble growth implies that bubble dynamics are not important for this flow. This accounts for the barotropic computation being almost identical to the dynamic computation. For flows nearing the critical regime, bubble dynamics become important and the dynamic and barotropic models obtain vastly different results.

Agreement of these models to the experimental pressure and liquid velocities is excellent, as it was also for the model of Ishii et al. The computed void fraction fares much worse. The only point of agreement is right in the throat itself, with the other points being considerably lower upstream of the throat and higher downstream of the throat. The considerably more complicated model of Ishii et al., which incorporates the relative motion of the phases, had reasonable success at matching the first and last experimental points, but significantly underestimated the void fraction at and immediately downstream of the throat.

\section{B. Unsteady flows with shocks}

Sandhu and Jameson ${ }^{19}$ performed experiments in a converging-diverging nozzle with equal inlet and outlet areas, and a throat area ratio of 0.132 . The nozzle diameter varied linearly between the transitions, which meant the area varied quadratically. In the implementation of the unsteady code, the function describing the throat area was constructed of Gaussian and error functions to ensure that it was infinitely differentiable, even at the transitions. The amount of smoothing at the transitions was kept to a minimum so as to have minimal impact on the flow.

Sandhu and Jameson used a surfactant to reduce bubble coalescence and hence maintain a large proportion of very fine bubbles. The surfactant would reduce the surface tension of the water, but in the absence of any data we use $S^{\prime}$ $=0.073 \mathrm{~N} / \mathrm{m}$, which is the value for water at $20^{\circ} \mathrm{C}$. Other flow conditions are, water with density and vapor pressure, $\rho_{L}^{\prime}=1000 \mathrm{~kg} / \mathrm{m}^{3}$ and $p_{v}^{\prime}=3.5 \mathrm{kPa}$, inlet pressure, $p_{0}^{\prime}$ $=214 \mathrm{kPa}$, and air bubbles with average equilbrium radius, $R_{0}^{\prime}=6 \times 10^{-5} \mathrm{~m}$. The inlet velocity was not specified in Sandhu and Jameson, but from a range of volume flow rates it was possible to determine that the velocity ranged from 1.1 to $3.1 \mathrm{~m} / \mathrm{s}$. It was found by trial and error with the steady code that an inlet velocity of $u_{0}^{\prime} \approx 2.27 \mathrm{~m} / \mathrm{s}$ resulted in choked flow. The above values resulted in a cavitation number, $\sigma$ $=81.6$ and Weber number, $W e=4.1$. In calculations we use an effective damping of $\delta_{D}=15.2$, which was determined to be "asymptotically small" according to the method discussed in Sec. III D.

Computations with different back pressures were performed. In each case as the back pressure was lowered the flow accelerated until at some instance the flow became choked and a bubbly shock wave formed in the diverging section of the nozzle. The position of the shock would oscillate about its steady-state position for a few cycles. Computations were carried out until it was clear where the final steady-state position of the shock was.

For these flows the maximum bubble growth was over 300 percent which results in $\sqrt{A_{\min }^{\prime}} / R_{\max }^{\prime}=20$. The continuum 

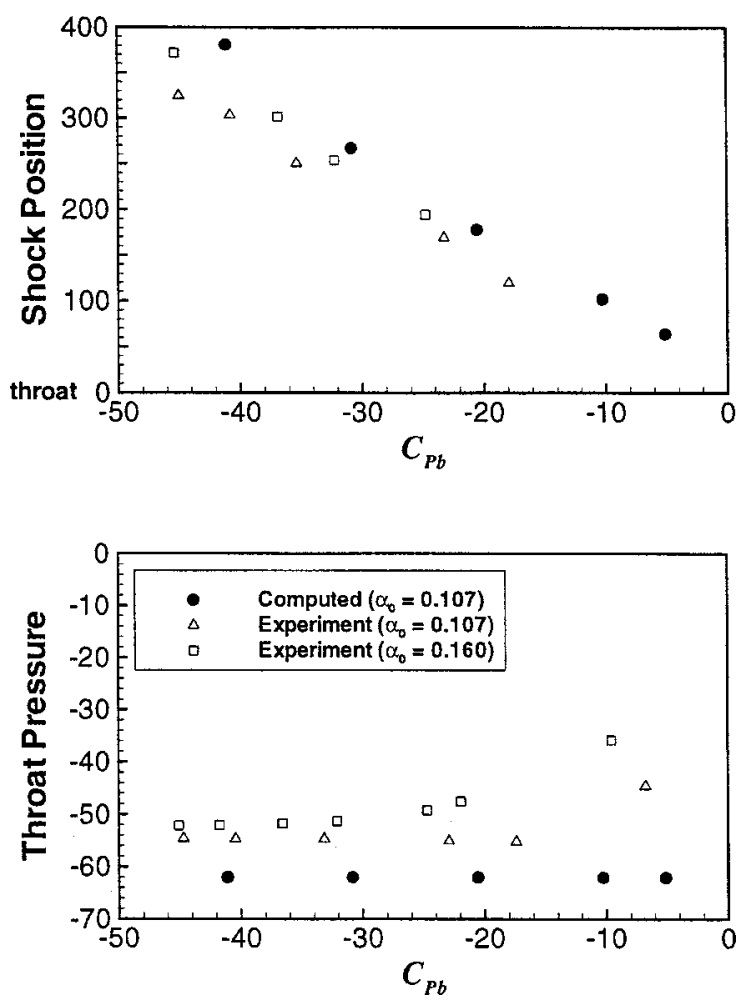

FIG. 10. Shock position and throat pressure as functions of back pressure for present computation and experimental observations of Sandhu et al. (parameters are $\sigma=81.6, W e=4.1, \delta_{D}=15.2$ ).

approximation is probably still valid, but it should be noted that the dilute gas-phase assumption is violated with void fractions as high as 25 percent being reached. Hence bubble-bubble interactions are important, and the RayleighPlesset equation should be modified to account for this. Nevertheless, the upper plot of Fig. 10 shows reasonable agreement of the computed steady-state shock position to the experimental observations presented in Fig. 4 of Sandhu and Jameson. The rightward shift of the computed points may be attributed to the friction losses in the experiment that are not accounted for in the model. To end up with a bubbly shock in a certain fixed position, the experiment would require a larger negative back pressure to overcome the additional frictional losses. Since the dissipation associated with the shock jump conditions is far greater than the viscous losses, it is reasonable to assume that the fractional effects do not significantly alter the flow field. We can here use the densities and velocities from the inviscid computation to estimate the pressure drop due to viscous losses in the experiment by assuming a fully developed turbulent pipe flow. For the data point at $C_{P_{b}} \approx-20$ in the upper plot of Fig. 10 we estimate the viscous losses in the experiment to be $\Delta C_{P_{\text {visc }}} \approx 4.0$. Hence if we were to include the friction losses in the model we would have to lower the back pressure an additional 4.0 units to achieve a shock in the same position. This corresponds to moving the data point 4.0 units to the left, which would then give good agreement with the experimental results.

The lower plot of Fig. 10 shows that the computed throat pressure is about ten percent larger than that measured ex-

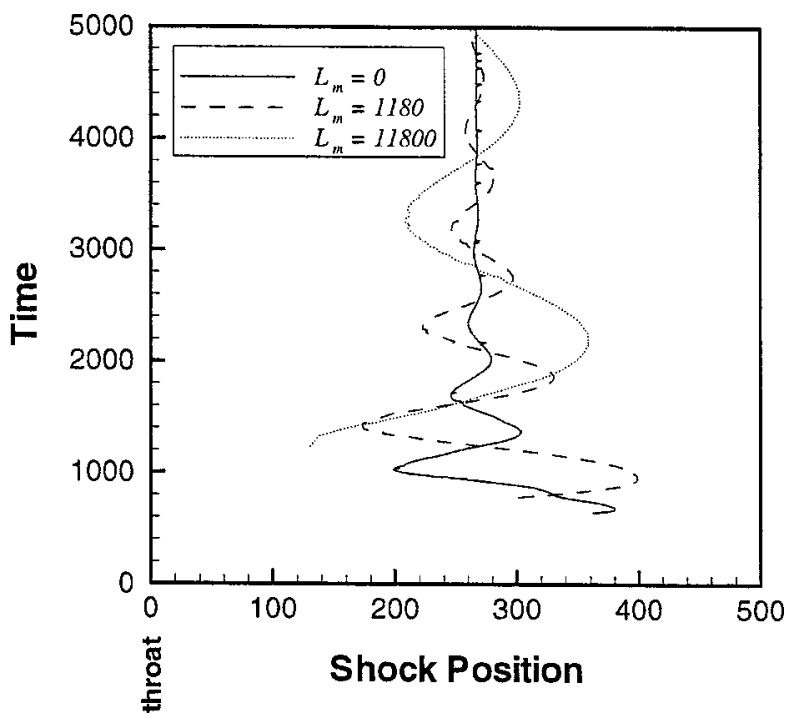

FIG. 11. Shock position versus time for different impedance lengths $\left(C_{P b}\right.$ $=-30.8$ ).

perimentally, and does not closely follow the upward trend on the right of the graph. These differences can again be attributed to the friction losses in the experiment that are not accounted for in the model. In the experiment a relatively small negative back pressure can result in a shockfree flow since there are appreciable friction losses to support the pressure gradient. Hence the experimental data points trend upwards on the right of the graph. The numerical model however only has losses in the bubble dynamics, so the same negative back pressure results in the flow accelerating until it chokes and a bubbly shock forms. Hence the throat pressure remains at the constant choked value.

Effect of impedance BC on shock position. In physical experiments there is usually a length of pipe (that may have valves and other apparatus) downstream of the nozzle section before the fluid exits into the atmosphere or reservoir. This has the effect of adding some impedance to the system, so that the back pressure is not accurately controlled immediately downstream of the nozzle section; instead it would tend to fluctuate about some mean value. To investigate the effect that this would have on the computed shock position an impedance $\mathrm{BC}$ was implemented at the downstream boundary. This was done, for illustrative purposes, using a simple force balance model at the boundary

$$
p_{N}-p(t)=\rho L_{m} \frac{d u_{N}}{d t}
$$

where $\rho$ is the average density of the bubbly mixture at the boundary, $L_{m}$ is a specified impedance length, $p(t)$ is the specified back pressure, and $p_{N}$ and $u_{N}$ are the pressure and velocity at the last grid point in the computational domain.

Figure 11 plots the time evolution of the shock position for three different impedance lengths. In each case the shock position exhibits the behavior of a damped oscillator. The final steady-state shock position and the initial amplitude of oscillation are independent of the impedance length. Initially 


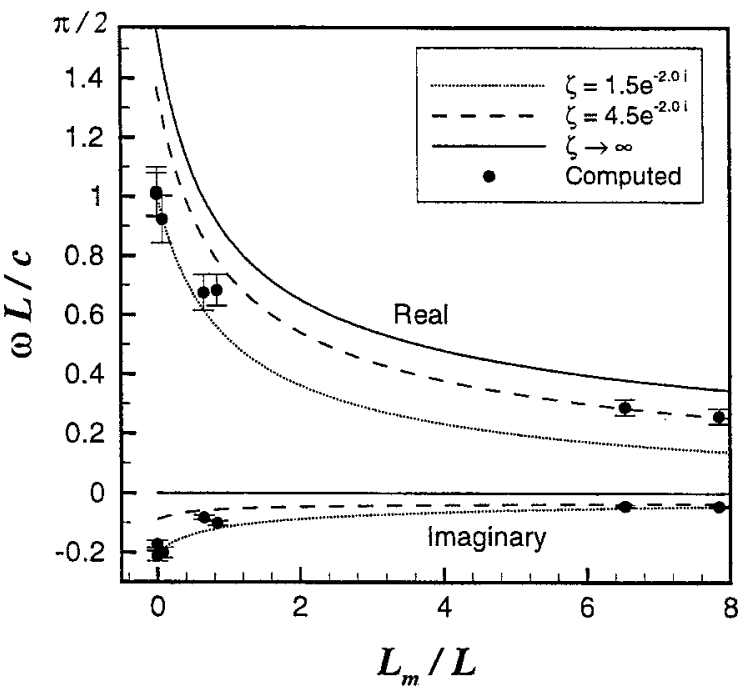

FIG. 12. Frequency of shock oscillation as a function of impedance length $\left(C_{P b}=-30.8\right)$.

the shock position oscillates back and forth about 200 bubble radii which, based on the average equilibrium bubble radius of the experiment, corresponds to $12 \mathrm{~mm}$. This is of the same order as the $2-5 \mathrm{~mm}$ observed in the experiment. As would be expected, the period of the transient oscillations increases with increasing impedance length.

The dependence of the oscillation frequency on impedance length can be explained by considering acoustic modes in the length of duct between the shock and the downstream boundary. Assuming that the length, $L$, and the linear (zero frequency) sound speed, $c$, between the bubbly shock and the downstream boundary are constant, and neglecting the flow velocity as being small compared to the sound speed, then this region is governed by the acoustic wave equations. The boundary conditions can be expressed in terms of the nondimensional complex impedance, $\zeta$

$$
\hat{p}=\rho c \zeta \hat{u} \text {. }
$$

Equation (31) directly yields $\zeta=-i \omega L_{m} / c$ as the value of impedance at the downstream boundary. It should be noted that Eq. (32) is an approximation for the far more complicated behavior of the bubbly shock; in reality there would be a complex dynamic interaction between the bubbly shock and an acoustic wave. Nevertheless we assume a complex value for the shock impedance, and then solve the resulting acoustic problem for a series of discrete complex frequencies. Figure 12 plots the lowest (fundamental) frequency as a function of impedance length for three assumed values of shock impedance. As expected these theoretical curves show that the real part of the frequency decreases as the impedance length is increased. Note that the normalized frequency is $\pi / 2$ for the case of infinite shock impedance and zero impedance length. This corresponds to the familiar resonant frequency for an open-closed tube.

Also plotted on Fig. 12 are some points obtained from computations. The real part of the frequency is obtained by applying a fast-Fourier transform to the time series of the shock position. (There are not enough cycles to allow use of an FFT for the two highest impedance lengths, so the frequency is computed by measuring the period of oscillation in Fig. 11.) The imaginary part of the frequency is computed from the ratio of amplitudes of successive peaks on Fig. 11. It is worth noting that the damping ratio is approximately constant for all values of impedance length, so that the attenuation per cycle is independent of impedance length. The error bars essentially indicate the resolution of the FFT. Estimates of the error due to not knowing the domain length and sound speed precisely were also made, but were about an order of magnitude smaller than the resolution of the FFT.

The acoustic theory correctly predicts the trend of decreasing real frequency with increasing impedance length for complex shock impedances with magnitudes ranging from near unity to infinity. In the limit of infinite shock impedance the theory results in an imaginary frequency of zero, in contrast to the computed data points. To obtain reasonable absolute agreement for both real and imaginary frequencies it is necessary to use a shock impedance with magnitude ranging from 1.5 to 4.5 and a complex phase of about -2 . If we were to further allow the magnitude of the shock impedance to be a function of frequency it is conceivable that we could obtain a single theoretical curve to match all the computed data points. In any case, the frequency of the shock oscillation is demonstrated to be governed by acoustic modes between the shock and the downstream boundary, and so in physical experiments the observed frequency would depend upon the experimental apparatus that existed between the shock and the pressure release surface.

\section{CONCLUSION}

An efficient and accurate numerical method has been developed for computing unsteady, quasi-one-dimensional, bubbly cavitating flows through converging-diverging nozzles. Four different flow regimes are shown to exist depending on the value of the back pressure. For small negative back pressures there exist steady state solutions with no shocks. As the back pressure is lowered the flow becomes choked, and a steady bubbly shock wave forms in the diverging section of the nozzle. For lower back pressures the bubbly shock wave begins to travel downstream in the diverging section of the nozzle. This unsteady bubbly shock wave is the correct solution in the regime where steady-state computations result in flashing solutions. Finally, for even lower back pressures, there exist underexpanded, steady-state solutions with no shocks.

Results are demonstrated to agree with barotropic models for those cases where bubble dynamics are not important, but show that in many instances that the neglect of bubble dynamics in the barotropic models cannot be justified. The computations show reasonable agreement with two sets of experiments; one where spatial variations of flow variables are measured in steady flows, and the other where throat pressure and shock position are measured for flows with bubbly shocks. The frequency of oscillation of the shock position is shown to be dependent on downstream impedance, and can be explained by considering acoustic modes in the region between the shock and downstream boundary. 
${ }^{1}$ R. F. Tangren, C. H. Dodge, and H. Seifert, "Compressibility effects in two-phase flow," J. Appl. Phys. 20, 637 (1949).

${ }^{2}$ C. E. Brennen, Cavitation and Bubble Dynamics (Oxford University Press, New York, 1995).

${ }^{3}$ Y.-C. Wang and C. Brennen, "One-dimensional bubbly cavitating flows through a converging-diverging nozzle," J. Fluids Eng. 120, 166 (1998).

${ }^{4} \mathrm{~L}$. van Wijngaarden, "On the equations of motion for mixtures of liquid and gas bubbles," J. Fluid Mech. 33, 465 (1968).

${ }^{5}$ L. van Wijngaarden, "One-dimensional flow of liquids containing small gas bubbles,” Annu. Rev. Fluid Mech. 4, 369 (1972).

${ }^{6} \mathrm{~A}$. Biesheuvel and L. van Wijngaarden, "Two phase flow equations for a dilute dispersion of gas bubbles in liquid,” J. Fluid Mech. 148, 301 (1984).

${ }^{7}$ C. F. Delale, G. H. Schnerr, and J. Sauer, "Quasi-one-dimensional steadystate cavitating nozzle flows," J. Fluid Mech. 247, 167 (2001).

${ }^{8}$ R. Ishii, Y. Umeda, S. Murata, and N. Shishido, "Bubbly flows through a converging-diverging nozzle," Phys. Fluids A 5, 1630 (1993).

${ }^{9}$ Y. Chen and S. D. Heister, "Modeling hydrodynamic nonequilbrium in cavitating flows," Trans. ASME 118, 172 (1996).

${ }^{10}$ A. Kubota, H. Kato, and H. Yamaguchi, "A new modelling of cavitating flows: a numerical study of unsteady cavitation on a hydrofoil section," J. Fluid Mech. 240, 59 (1992).

${ }^{11}$ T. Colonius, C. E. Brennen, and F. d'Auria, "Computation of shock waves in cavitating flows," ASME/FED Third International Symposium on Numerical Methods for Multiphase Flow, June 1998.

${ }^{12}$ T. Colonius, F. d'Auria, and C. E. Brennen, "Acoustic saturation in bubbly cavitating flow adjacent to an oscillating wall," Phys. Fluids 12, 2752 (2000).

${ }^{13}$ E. Hairer and G. Wanner, Solving Ordinary Differential Equations II, rev. ed. (Springer, New York, 1996).

${ }^{14}$ W. H. Press, S. A. Teukolsky, W. T. Vettering, and B. P. Flannery, Numerical Recipes in FORTRAN: The Art of Scientific Computing, 2nd ed. (Cambridge University Press, Cambridge, 1994).

${ }^{15}$ Y.-C. Wang, "Stability analysis of one-dimensional steady cavitating nozzle flows with bubble size distribution," 1999 ASME/JSME FED Summer Meeting, September 1999.

${ }^{16}$ M. Watanabe and A. Prosperetti, "Shock waves in dilute bubbly liquids," J. Fluid Mech. 274, 349 (1994).

${ }^{17}$ M. Kameda and Y. Matsumoto, "Shock waves in a liquid containing small gas bubbles," Phys. Fluids 8, 322 (1996).

${ }^{18}$ Y. Matsumoto and M. Kameda, "Propagation of shock waves in dilute bubbly liquids," JSME Int. J., Ser. B 39, 264 (1996).

${ }^{19}$ N. Sandhu and G. J. Jameson, "An experimental study of choked foam flows in a convergent divergent nozzle," Int. J. Multiphase Flow 5, 39 (1979). 